\title{
Mecanismos y procedimientos aplicados al análisis del aliento para diagnóstico de diferentes enfermedades utilizando métodos multisensoriales
}

\author{
Juan Alberto Borré Lara1'., Cristhian Manuel Durán Acevedo²., Oscar Eduardo \\ Gualdron Guerrero³.
}

1. Universidad de Pamplona, Pamplona, Colombia, Facultad de Ingenierías y Arquitectura. Grupo de investigación Sistemas multisensoriales y reconocimiento de patrones.

2. Universidad de Pamplona, Pamplona, Colombia, Facultad de Ingenierías y Arquitectura. Grupo de investigación Sistemas multisensoriales y reconocimiento de patrones.

3. Universidad de Pamplona, Pamplona, Colombia, Facultad de Ingenierías y Arquitectura. Grupo de investigación Sistemas multisensoriales y reconocimiento de patrones.

\section{Resumen}

En el presente estudio se describe un análisis general de los distintos mecanismos y procedimientos aplicados al análisis del aliento para diagnostico precoz de diferentes enfermedades.

En gran parte de estos métodos se da a conocer que la mayoría del contenido del aliento exhalado está formado por una gran variedad de compuestos (por ejemplo: nitrógeno, oxígeno, de dióxido de carbono, agua, gases inertes, entre otros). Las fuentes de estos volátiles son los componentes atmosféricos que se originan a partir de muchos disolventes y productos a base de petróleo, y otros compuestos orgánicos volátiles que se generan como resultado de los procesos bioquímicos del cuerpo.

En la mayoría de los estudios encontrados describen los diferentes sistemas de muestreo y dispositivos de medición, de los cuales en este estudio se referencian los que son aplicados a enfermedades terminales (por ejemplo, el cáncer).

Palabras clave: Sistemas de muestreo, aliento exhalado, enfermedades, compuestos volátiles, técnicas de análisis.

\section{Mechanisms and procedures applied to breath analysis for diagnostic various diseases using multisensorials methods}

\begin{abstract}
In the present study an overview of the various mechanisms and applied techniques to the analysis of encouragement for early diagnosis of different diseases is described.

In many of the procedures is disclosed that most of the content of exhaled breath is nitrogen, oxygen, carbon dioxide, water and inert gases. These sources are atmospheric volatile
\end{abstract}


components originating from many solvents and petroleum-based products, anfgd volatile organic compounds that are generated as a result of biochemical body processes. The classes of volatile organic compounds, which can be measured in exhaled breath, are coupled with oxygen, sulfur, and unsaturated and saturated hydrocarbon compounds containing nitrogen. However, those tests are not ideal because they are expensive, require expertise, and they need to collect and transport the contents of encouragement to devices.

Keywords: Sampling systems, exhaled breath, diseases, volatiles, analysis techniques

*Para citar este artículo: Borré Lara JA; Durán Acevedo CM; Gualdròn Guerrero OE. Mecanismos y procedimientos aplicados al análisis del aliento para diagnóstico de diferentes enfermedades utilizando métodos multisensoriales. Revista Bistua.2016.14(1):99-111

+ Autor para el envió de correspondencia y la solicitud de las separatas: Gualdròn Guerrero OE. Grupo de Investigación Sistemas multisensoriales, Departamento de Electrónica .Universidad de Pamplona, Colombia... email: oscar.gualdron@unipamplona.edu.co 


\section{Introducción}

En recientes estudios el análisis de la respiración a través del aliento se ha propuesto como un método complementario, conveniente y seguro en comparación con las técnicas de diagnóstico tradicionales ${ }^{1}$. Es más, ha sido demostrado científicamente que los perros pueden oler el cáncer de pulmón, esto debido a que ante la presencia de diferentes grados de tumoración, ellas van acompañada por cambios genéticos y/o proteínicos que conducen a un efecto de per oxidación de las membranas celulares conllevando a la emisión de compuestos orgánicos volátiles. Los biomarcadores derivados de la respiración pueden proporcionar más seguridad, mayor sensibilidad y especificidad de los estudios tradicionales, arriesgándonos a decir que se podría catalogar como un método novedoso de diagnóstico no invasivo, siendo una alternativa de gran interés para la detección temprana de las enfermedades. Haciendo un repaso al estado del arte actual relacionado con la problemática mencionada anteriormente, se encuentra que han habido avances muy interesantes en el desarrollo de métodos para la detección de enfermedades a través del análisis de aliento, principalmente para enfermedades respiratorias como son el cáncer del pulmón, asma, EPOC, entre otras $^{2,3,4}$, donde se encuentran diferencias estadísticamente significativas al comparar los patrones de componentes orgánicos volátiles (COVs) de personas con o sin las enfermedades mencionadas, y más allá, de diferentes grados de la enfermedad (pacientes graves, pacientes moderados).

Peng $G$, et $a l^{5}$, presentan también resultados relacionados con otro tipo de enfermedades como son las de cáncer de próstata, cáncer colon-rectal, de mama, de cabeza y garganta, esclerosis múltiple, entre otros, usando arreglos simples de nanosensores. Obteniendo resultados interesantes no solo para diferenciar entre pacientes saludables y pacientes con cáncer sino distinguir entre patrones de aliento de diferentes tipos de cáncer con el mismo análisis estadístico.Dichos investigadores han desarrollado a tal respecto un sistema basado en una agrupación de sensores ('MNA-NOSE) que se valen de nanoparticulas metálicas $\mathrm{y} / \mathrm{o}$ nanotubos de carbono funcionalizados con moléculas orgánicas con propiedades hidrofóbicas(para contrarrestar el gran contenido de agua en el aliento) para detectar y analizar pequeñas concentraciones de partículas orgánicas volátiles que se desprenden de las células y son regularmente emitidas al respirar, con concentraciones por debajo de una millonésima parte, es decir, los nanosensores experimentan cambios al ser expuestos a los COVs del aire respirado que se traducen en patrones. Estos patrones de COVs pueden ser usados como biomarcadores no invasivos de varios procesos 
otras condiciones menos severas), en

bioquímicos que se dan en las enfermedades.Por otro lado ${ }^{6}$, presentan un método novedoso basado en testeo del aliento alveolar exhalado para identificar cáncer de mama precoz en condiciones benignas, malignas y en estado saludable, tomando como referencia 36 voluntarios usando una nariz electrónica a nano escala analizando dicha información con técnicas de análisis de componentes principales, ANOVA y maquinas de soporte vectorial $^{7}$, igualmente usaron muestras de aliento exhalado para diagnosticar cáncer de garganta empleando muestras de 87 voluntarios basándose en cinco sensores de nano partículas de oro, tendiendo la particularidad en este estudio, de realizar en paralelo un análisis de la composición química de las muestras a través de cromatografía de gases en conjunto con espectrometría de masas, usando 40 muestras recopiladas, destacándose seis compuestos químicos que hacen la diferencia entre pacientes sanos y con cáncer de garganta. En los últimos años se ha direccionado estos estudios para explorar el uso del aliento exhalado en la detección temprana de enfermedades gástricas $^{8}$, en particular, el cáncer gástrico en diferentes estados, por ello ${ }^{9}$, presentan resultados que permiten distinguir entre cáncer gástrico con respecto a condiciones gástricas benignas usando sensores basados en nanomateriales, tomando como referencia muestras de 130 pacientes provenientes de hospitales de china y letonia con diferentes condiciones este trabajo se emplearon modelos predictores como son el análisis de factor discriminante (DFA), para construir los clasificadores. Igualmente se realizaron análisis químicos usando cromatografía de gases, generándose resultados interesantes a la hora de clasificar pacientes con cáncer con respecto a otras condiciones, diferenciación entre grados de la enfermedad (temprana y tardía) y finalmente ante la presencia del $\mathrm{H}$ pylori. El grupo de investigadores también realizaron un estudio sobre los biomarcadores volátiles en el aliento de dos poblaciones con elevada incidencia de problemas gástricos, pero con rasgos genéticos y nutricionales completamente distintos (China y Letonia). Encontrándose que sí hay un biomarcador común para el cáncer gástrico, y algunos compuestos para la úlcera, así como biomarcadores encontrados solo en una de las dos poblaciones.

\section{SISTEMA DE MEDICION}

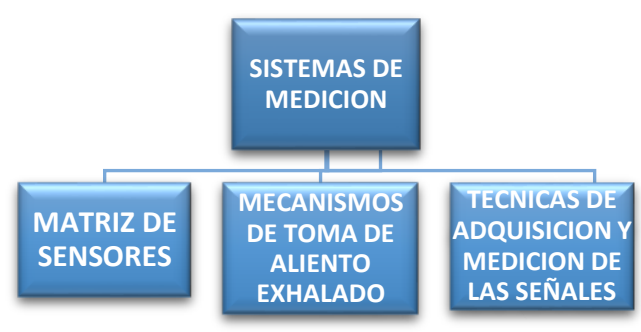

Fig.1 Partes del sistema de medición 


\section{Matriz de sensores}

Los compuestos volátiles emitidos por el aliento exhalado de las personas son por lo general: el nitrógeno, oxígeno, dióxido de carbono, agua y gases inertes. Las fuentes de éstos volátiles son los componentes atmosféricos que se originan a partir de muchos disolventes y productos derivados del petróleo, y los compuestos orgánicos volátiles que se generan como resultado de los procesos bioquímicos del cuerpo. Los principales compuestos orgánicos volátiles que se pueden medir en el aliento exhalado son los hidrocarburos insaturados $y$ saturados, junto con oxígeno, azufre, y compuestos que lo contienen ${ }^{10}$. En la medición de los compuestos exhalados es necesario mencionar el avance de las matrices de sensores el cual es uno de los componentes principales en el análisis de las muestras, por consiguiente se describen a continuación algunos de los sensores más utilizados como son la de sensores colorimétricos, matriz de nanotubos de carbono, sensores de superficie de onda acústica, sensores de nano partículas de oro y sensores de fibra óptica. Para la descripción de un diagnóstico de cáncer de pulmón a través del análisis de aliento exhalado, Mazzon y colaboradores evidencian el uso de un conjunto de sensores colorimétricos, el cual se compone de 36 puntos como se muestra en la Fig. 2, cada uno con un compuesto sensible químicamente diferente, que responde a los compuestos reactivos con un cambio de color.

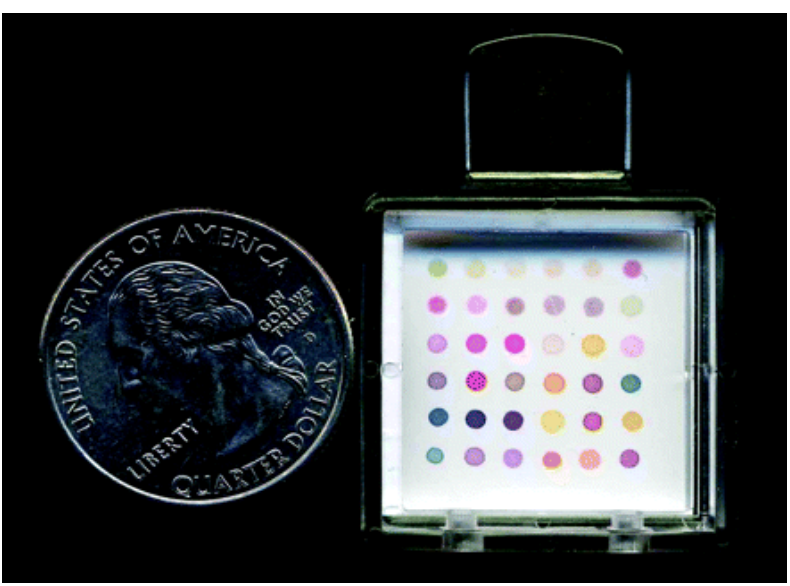

En otro estudio se reportó el diseño de nariz electrónica, que consta de cinco sensores de gases desarrollados mediante compuestos de polímeros conductivos. Esta nariz electrónica desarrollada detecta nueve vapores de disolventes orgánicos (isopropanol, tetrahidrofurano, diclorometano, nheptano, ciclohexano, metanol, etanol, agua y tolueno), así como biomarcadores para la detección de cáncer de pulmón en el análisis del aliento $^{11}$.

Otro sensor utilizado para la detección de compuestos del aliento, es el desarrollado con nanotubos de carbono de pared simple revestidos con materiales orgánicos no poliméricos, estos fueron usados para detectar cáncer de pulmón con el objetivo de diferenciar entre los COV's (Compuestos Orgánicos Volátiles) de pacientes con cáncer de pulmón con respecto a pacientes sanos ${ }^{12}$; dichos sensores fueron fabricados con las tecnologías de micro-mecanizado y funcionan por el efecto de campo de polarización y de ionización del 
104

dispositivo. Estos dispositivos presentan diferentes propiedades tales como: pequeña relación de punta, alta relación de aspecto y una elevada conductividad eléctrica, su vez, estos sensores presentaron buena sensibilidad en la detección de la respiración débil del paciente.

En otros hallazgos se evidencia que un sensor de nano partículas de oro podría diferenciar entre los pacientes sanos y pacientes que sufren de cáncer, organizados una matriz hecha a medida de los sensores de reacción cruzada basada en nano partículas de oro funcionalizadas orgánicamente discriminan entre COV del aliento de pacientes sanos y de pacientes que sufren de cáncer de pulmón, mama, colorrectal y cáncer de próstata.

Por otra parte, en el trabajo realizado por Peng y colaboradores se describe la capacidad de estos sensores PNB para distinguir entre los patrones de respiración de los diferentes tipos de cáncer en el mismo análisis de patrones, independientemente de la edad, sexo, estilo de vida y otros factores de confusión ${ }^{13}$.

Un sensor de fibra óptica para aplicaciones médicas fue desarrollado con el fin de realizar un diagnóstico clínico ${ }^{14}$. El objetivo fue determinar ocho compuestos orgánicos volátiles en el aliento humano, (etano, pentano, heptano, octano, decano, benceno, tolueno y estireno). Las variaciones en la potencia óptica se detectaron como resultado de los cambios inducidos por las moléculas del analito a través de la sensibilidad del índice de refracción de la película polimérica. Los cambios reflejados en la potencia óptica fueron proporcionales a la cantidad del analito. El dispositivo obtuvo una precisión adecuada y con un alto grado de linealidad para el modelo de calibración y detección de compuestos.

Este sensor mostró características ventajosas de respuesta en tiempo real y bajos costos de instrumentación, además de mostrar un rendimiento analítico equivalente al análisis del aliento por cromatografía de gasespectrometría de masas, que se utiliza como método de referencia.

Como conclusión, para Wang y colaboradores las opciones más adecuadas a ser usadas en un sistema olfato electrónico entre las tecnologías de detección de gas disponibles son los sensores de ondas acústicas y micro balanza de cristal de cuarzo ${ }^{15}$.

\section{Mecanismos de toma de aliento exhalado}

En este apartado se describen algunos mecanismos desarrollados en los últimos años, los cuales han permitido acondicionar la muestra desde el aliento exhalado. Estos se encargan de tomar el aliento y distribuirlo a diferentes fases para su purificación, absorción y almacenado para luego analizar la muestra. En la actualidad estos dispositivos se quieren de forma que el resultado de los estudios se genere de forma instantánea y así el paciente puede obtener su resultado y gracias a un examen no invasivo. 


\section{Sistema de muestreo y capnografo}

\section{Dispositivo Pneumopipe}

El análisis del aliento exhalado comúnmente se basa en primera medida por la obtención del aliento por medio de un mecanismo de toma para luego ser almacenado en un precocentrador y después ser analizado por técnicas de adquisición.

Por tal razón en la Fig. 3 se observa el esquema del sistema "Pneumopipe", un dispositivo novedoso para toma del aliento desarrollado por Giorgio Pennazza y colaboradores el cual es un cartucho-adsorbente que permite un muestreo continuo de aire exhalado ${ }^{16}$. La cámara inferior del dispositivo es para la exhalación de aire que se dirige hacia la cámara superior.

El sistema en forma general se compone de una mufla, la cual mantiene una temperatura constante en el centro del cartucho. Este sistema se compone de válvulas que se cierran en el momento de una inspiración y se abren en el momento de una exhalación, para así garantizar que la muestra de aliento exhalada no se contamine con el ambiente.

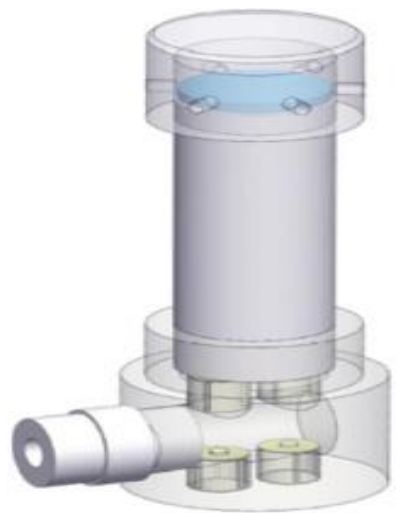

Fig. 3 Panorama general de la Pneumopipe [16] 105
En otros estudios sobre la detección de enfermedades por medio del aliento $\mathrm{R}$. Fernandez y colaboradores diseñaron un sistema muy sencillo que contiene un sensor de $\mathrm{CO} 2$ en la corriente principal de la línea conectada a un capnógrafo y una jeringa de vidrio de $100 \mathrm{ml}$ se acopló a la tubería usando una llave de paso de 3 vías como se muestra en la Fig. $4^{17}$. Se recogieron muestras de tres a cuatro respiraciones por cada jeringa de $100 \mathrm{ml}$, y cuatro repeticiones de los mismos se tomaron para cada sujeto. Las jeringas utilizadas en este sistema son de vidrio ya que las muestras no tienen volátiles contaminantes.

Fig 4. Esquema de muestreo [17]

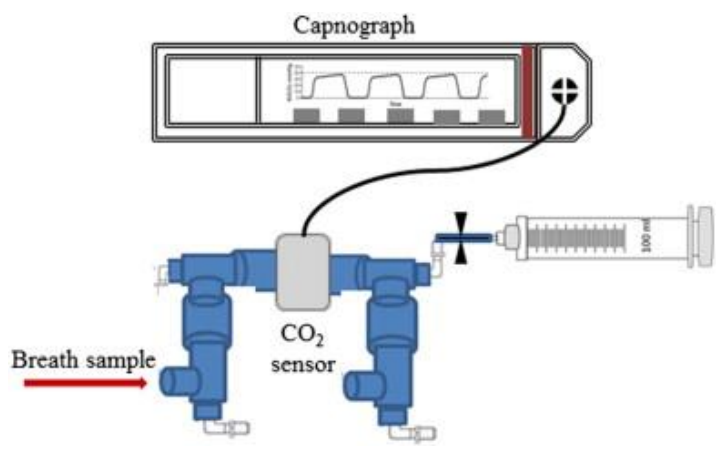

\section{BufferedEnd-Tidal (BET)}

En estudios por JensHerbig y colaboradores se describe una nueva herramienta llamada BET, ver Fig. 5, dispositivo en línea donde el sujeto inhala y exhala a través de un tubo de muestreo ${ }^{18}$. En nuestro enfoque, una sola exhalación se requiere por minuto para el muestreo y para el sujeto de prueba de lo contrario puede

Bistua Revista de la Facultad de Ciencias Basicas .Universidad de Pamplona. ISSN 0120-4211 

normal, reduciendo el riesgo de hiperventilación. Para validar el nuevo método de muestreo BET se hicieron mediciones comparativas realizadas con el muestreo en línea directa utilizando de transferencia de protones-reacción espectrometría de masas.

Fig 5. Dispositivo BET [18]

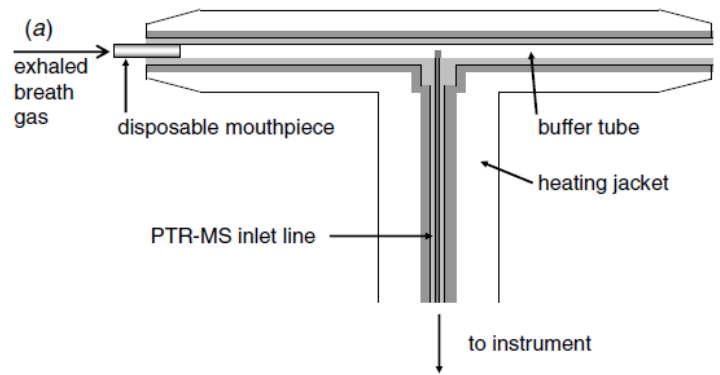

Técnicas clásicas de adquisición y medición de señales

Actualmente las técnicas de GC-MS (Gas Chromatography

MassSpectrometry), se presentaron como las técnicas dominantes, solo o en combinación con otras técnicas, como la desor-térmica automatizada, espectroscopia láser, espectrometría de masas (SIFT-MS) y espectroscopia de infrarrojo medio $(\mathrm{MIR})^{19}$.

\section{Cromatografía de gases}

En cromatografía de gases (GC), la muestra se inyecta en la fase móvil, la cual es un gas inerte. En esta fase, los distintos componentes de la muestra pasan a través de la fase estacionaria que se encuentra fijada en una columna. Actualmente, las más empleadas son las columnas capilares ${ }^{19}$.La columna se encuentra dentro de un horno con temperatura programada. La velocidad de migración de cada componente será función de su distribución entre la fase móvil y la fase estacionaria. Cada soluto presente en la muestra tiene una diferente afinidad hacia la fase estacionaria, lo que permite su separación: los componentes fuertemente retenidos por esta fase se moverán lentamente en la fase móvil, mientras que los débilmente retenidos lo harán rápidamente. Un factor clave en este equilibrio es la presión de vapor de los compuestos (en general, a mayor presión de vapor, menor tiempo de retención en la columna). Como consecuencia de esta diferencia de movilidad, los diversos componentes de la muestra se separan en bandas que pueden analizarse tanto cualitativa como cuantitativamente mediante el empleo de los detectores seleccionados.

Existen tres técnicas básicas de inyección de muestras (líquidas o gaseosas) en columnas capilares: split, split-less y oncolumn. Las dos primeras consisten en inyectar y vaporizar la muestra en una cámara de vaporización. El sistema split desvía la mayor parte de la muestra fuera del sistema cromatográfico y envía sólo una pequeña fracción a la columna. El método split-less dirige toda la muestra a la columna, por lo que resulta más adecuado para el análisis de trazas o de componentes muy volátiles. La inyección oncolumn se lleva a cabo en frío, eliminando la etapa de vaporización que podría producir la 
descomposición de los compuestos termolábiles.

\section{Espectrometría de masas}

La espectrometría de masas (MS) es una de las técnicas analíticas más completas que existen ${ }^{19}$. Recientemente, esta técnica se utiliza no sólo en investigación, sino también en análisis de rutina de los procesos industriales, en control de calidad, etc.

Sus principales cualidades son:

- Capacidad de identificación de forma prácticamente inequívoca, ya que proporciona un espectro característico de cada molécula.

- Cuantitativa: permite medir la concentración de las sustancias.

- Gran sensibilidad: habitualmente se detectan concentraciones del orden de ppm o ppb y en casos específicos se puede llegar hasta ppt e incluso ppq.

- Universal y específica.

- Proporciona información estructural sobre la molécula analizada.

- Es una técnica rápida: se puede realizar un espectro en décimas de segundo, por lo que puede monitorizarse para obtener información en tiempo real sobre la composición de una mezcla de gases.

Dentro del espectrómetro de masas, se procede a la ionización de la muestra mediante diferentes métodos. El sistema de ionización más frecuente es el de impacto electrónico que bombardea las moléculas con electrones de una cierta energía,

Bistua Revista de la Facultad de Ciencias Basicas .Universidad de Pamplona. ISSN 0120-4211 capaces de provocar la emisión estimulada de un electrón de las moléculas y así ionizarlas. Esta técnica constituye una identificación prácticamente inequívoca del compuesto analizado. El espectro de masas puede almacenarse en la memoria del ordenador para compararse con los espectros de una colección de espectros (o librería) y proceder a su identificación.

\section{Reconocimiento de patrones}

El procesamiento de datos en las narices electrónicas es una de las partes más importantes ya que es donde se identifican y comparan los datos obtenidos, para este procedimiento existen varias técnicas, denominadas algoritmos de "reconocimiento de patrones" o "técnicas de inteligencia artificial" y se pueden definir como los procesos matemáticos que transforman los datos originales de las medidas en información útil para el usuario final ${ }^{20}$. Métodos paramétricos: Las técnicas paramétricas comúnmente se relacionan a una búsqueda estadística basada en la suposición de que la totalidad de los datos de entrada pueden ser descritos por una función densidad probabilística. Estas técnicas intentan encontrar una relación matemática subyacente entre el sistema de entrada (señales de los sensores) y sus salidas (clases 0 descriptores).

Métodos no paramétricos: los métodos no paramétricos no tienen en cuenta la 
función de densidad probabilística para los datos de entrada y su aplicación es más genérica. Dentro de esta clasificación nos encontramos con las redes neuronales artificiales y los sistemas expertos.

Supervisado: en un método de aprendizaje supervisado, un conjunto de datos de entrada conocido es sistemáticamente presentado al sistema, datos que son clasificados de acuerdo a los descriptores o clases determinadas previamente, y así, en una segunda etapa de identificación, datos de entrada no conocidos o nuevos se prueban con un conjunto de validación para mirar hasta qué punto los métodos supervisados son capaces de predecir las correspondientes clases.

\section{weighted digital analysis (WDA)}

WDA es un método matemático para el desarrollo de un algoritmo de diagnóstico que genera una puntuación a la función discriminatoria ${ }^{21}$. El valor de este marcador predice la pertenencia de 1 a 2 grupos. Cada variable de diagnóstico que se emplea en el algoritmo tiene tres parámetros.

Signo: Esto puede ser positivo $(+1)$ o negativo (-1). Si el signo es positivo, indica que la enfermedad es menos probable. Por el contrario, si su signo es negativo, indica que la enfermedad es más probable.

Peso. Este valor indica la contribución relativa de cada variable de diagnóstico para la función discriminatoria, es decir, cuanto mayor sea su peso, mayor es su importancia.
108

Valor de corte. Esto determina si es o no una variable de diagnóstico. Contribuye a la puntuación de la función discriminatoria. La contribución de una variable de diagnóstico (peso) se añadirá a la puntuación de la función discriminatoria sólo si dicho valor es superior al valor de corte.WDA genera una función discriminante para predecir la pertenencia a la enfermedad mediante la determinación del peso, un valor de corte, y un signo para cada variable. El peso de cada variable fue el área bajo la curva (AUC). El signo ( \pm ) se utilizó para invertir la variable predictiva si un valor es bajo indica una mayor probabilidad de enfermedad. Cuando se emplea para predecir la presencia de una enfermedad en un paciente en particular, la función discriminante se determina como la suma de los pesos de todas las variables predictivas que excedieron sus valores de corte.

\section{Support vector machine (SVM)}

El principio de esta familia de algoritmos consiste en construir un hiperplano para separar los datos de entrenamiento en diferentes clases, procurando que los vectores que pertenecen a las distintas clases se encuentran en distintos lados del hiperplano y que la mínima distancia entre los vectores y el hiperplano sea el máximo posible ${ }^{21}$. Si los datos no son linealmente separables se busca proyectar los datos del espacio de entrada en un espacio de mayor dimensión llamado espacio de características de modo que los datos se vuelvan linealmente separables. 


\section{$k$ nearest neighbour (kNN)}

Este algoritmo compara los datos de entrada con un conjunto existente de datos de entrenamiento mediante el cálculo de una distancia métrica. Los vecinos de los datos de entrada son los puntos de datos $k$ con la menor distancia. Las clases de los datos de entrada se determinan con la mayoría de los puntos de datos en el sector ${ }^{22}$.

\section{Bayesian networks (BN)}

Un trabajo reciente en el aprendizaje supervisado ha demostrado que un clasificador bayesiano sorprendentemente simple con un fuerte hipótesis de independencia entre las características, llamado sencillo de Bayes $^{23}$.

\section{Conclusiones}

En este artículo se estudiaron y analizaron diferentes mecanismos y procedimientos que se han ido aplicando en los últimos años en la detección de enfermedades a través del aliento. En algunos de estos estudios se encontraron aplicaciones desarrolladas a la detección del cáncer (pulmón y próstata), donde se ha demostrado que hay biomarcadores en el aliento que pueden dar un indicador de la presencia de la enfermedad.

Entre los sistemas de muestreo estudiados, se puede concluir que el BioVOC es más viable debido a que los pacientes a los que se len a realizar las pruebas, no deberán hacer un lavado previo o purga de los pulmones, a comparación con el sistema compuesto con otro método usando bolsas Mylar. Por otro lado con este nuevo sistema no

\section{9}

es necesario utilizar Nitrógeno puro $99.995 \%$, ya que la limpieza se hará con agua destilada y de una manera más flexible al usuario. Otro aspecto es la robustez del dispositivo BioVOC en comparación al sistema compuesto por bolsas Mylar.

\section{Agradecimientos}

Los integrantes del trabajo realizado agradecen el apoyo dado por COLCIENCIAS, al proyecto con código 112165741516 el cual fue financiado para el desarrollo de cada una de las diferentes actividades.

\section{Referencias bibliogràficas}

1. Martinez P, Kohler M, Zenobi R. (2013). Monitoring diurnal changes in exhaled human breath, Analytical chemistry. v. 85 , p. 369-373.

2. Ehmann R, Boedeker E, Friedrich, Sagert J, (2012). Canine scent detection in the diagnosis of lung cancer: revisiting a puzzling phenomenon, European respiratory journal. v.39 N. 3, p. 669-676.

3. Duran C, Velazquez A, Gualdron $O$, (2012). Electronic Nose To Detect Patients with COPD From Exhaled Breat, revista Ingeniería y desarrollo, v. 30, N. 2. p. 43159.

4. G Peng, M Hakim, YY Broza, S Billan, $R$ Abdah-Bortnyak, (2009). Detection of lung, cancers from exhaled breath. Nature nanotechnology. v. 4, p. 669-673.

5. G Peng, Hakin M, Broza $Y$, Billan $S$, Kuten A, (2010). Detection of lung, breast, colorectal and prostate cancers from exhaled breath using a single array of nanosensor. British journal of cancer, v. 103 , p. $542-551$. 
6. Shuster G, Gallimidi Z, Heyman A. (2011) Classification of breast cancer precursors through exhaled breath. Breast cancer research, v.126, p.791-796.

7. Hakim M, Billan S, Tisch U, Peng G, (2011). Diagnosis of head and neck cancer from exhaled breath. British journal of cancer, p.1-7.

8. Haitham A, Leja M, Haick H, (2013) Volatile Markers Can Discriminate Between Gastric Cancer and Benign Conditions, Gastroenterology, v. 144, I. 5, S. 1, p. S-353.

9. Broza $Y$, lonescu R, Tisch U, Ding L, (2013). A nanomaterial- based breath test for distinguishing gastric cáncer from bening gastric conditions. British journal of cancer $p$. 1-10.

10. Mazzone P, Hammel J, Jie Na, Czich C, (2007). Diagnosis of lung cancer by the analysis of exhaled breath with a colorimetric sensor array. Thorax. v. 62. P.565-568.

11. M. Castro, B. Kumar, J.F. Feller. (2011). Novel e-nose for the discrimination of volatile organic biomarkers with an array of carbon nanotubes (CNT) conductive polymer nanocomposites (CPC). Sensors Elsevier B.

12. Pen G, Trock E, Hossam Haick, (2008). Detecting Simulated Patterns of Lung Cancer Biomarkers by Random Network of Single Walled Carbon Nanotubes Coated with Nonpolymeric Organic Materials. American Chemical Society.

13. Bartosz, Wyszynski, Nakamoto, L. (2009). Biological and Artificial Olfaction: Biomimetic Quartz Crystal Microbalance Odor Sensors, Institute of Electrical Engineers of Japan.

14. G Peng, M Hakim, YY Broza, S Billan, (2010). Detection of colorectal, and prostate cancers from exhaled breath using a single array of nanosensors, Cancer Research UK.
110

15. Lurdes I.B. Silva, Ana C. Freitas, Teresa A.P. Rocha-Santos, (2010). Breath analysis by optical fiber sensor for the determination of exhaled organic compounds with a view to diagnostics, Elsevier B.V.

16. Pennazza G, Santonicoa, M, Antonelli R. (2014). Measure chain for exhaled breath collection and analysis: A novel approach suitable for frail respiratory patients, Elsevier B.V.

17. R. Fernández del Río, M.E. O'Hara, A. Holtb. (2015). Volatile Biomarkers in Breath Associated With Liver Cirrhosis Comparisons of Pre- and Post-liver Transplant Breath Samples, Elsevier B.V.

18. Jens Herbig, Thorsten Titzmann, Jonathan Beauchamp. (2008). Buffered end-tidal (BET) sampling-a novel method for real-time breath-gas analysis. Journal of breath research.

19. M.C. Gutiérrez, M. Droguet, (2002). La cromatografía de gases y la espectrometría de masas: Identificación de compuestos causantes de mal olor. Boletín Intexter (U.P.C.)

20. Gualdron O, (2006). Desarrollo de diferentes métodos de selección de variables para sistemas multisensoriales, Tesis Doctoral, Universidad Rovira i Virgili.

21. Phillipsa M, Altorkic N, Austind J, (2008). Detection of lung cancer using weighted digital analysis of breath biomarkers, Elsevier B.V.

22. Yekbun Adiguzel, HalukKulah. (2014). Breath sensors for lung cancer diagnosis, Elsevier B.V.

23. Nir Friedman, Geiger D, Goldszmidt, M. (1997). Bayesian Network Classifiers. Machine Learning v. 29. p. 131-163. 
111

Juan Alberto Borré Lara. Ingeniero Mecatrònico, Universidad de Pamplona. Magister en Controles Industriales. Universidad de Pamplona.

Cristhian Manuel Durán Acevedo. Ph.D. Profesor Asociado. Facultad Ingenierías y Arquitectura. Grupo de Investigación Sistemas Multisensoriales. Investigador Asociado

Oscar Eduardo Gualdron Guerrero. Ph.D. Profesor Asociado. Facultad Ingenierías y Arquitectura. Grupo de Investigación Sistemas Multisensoriales.

Investigador Asociado 